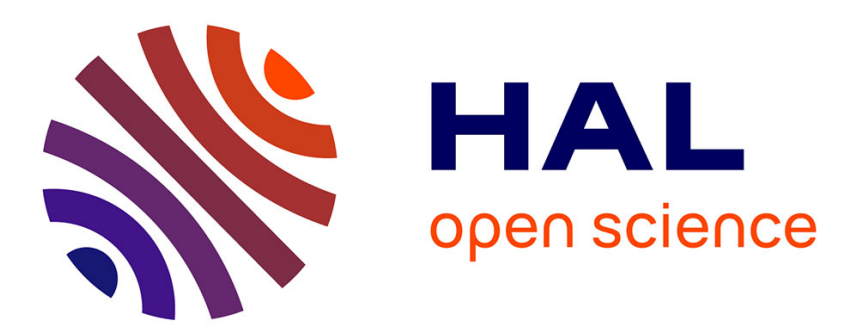

\title{
La première pétition en copte: révision de SB Kopt. IV 1709
}

\author{
Alain Delattre, Jean-Luc Fournet
}

\section{To cite this version:}

Alain Delattre, Jean-Luc Fournet. La première pétition en copte: révision de SB Kopt. IV 1709. Chronique d'Egypte; bulletin periodique de la Fondation egyptologique reine Elisabeth, 2018, 93, p. 167-186. 10.1484/J.CDE.5.116103 . hal-01990940

\section{HAL Id: hal-01990940 https://hal.science/hal-01990940}

Submitted on 23 Jan 2019

HAL is a multi-disciplinary open access archive for the deposit and dissemination of scientific research documents, whether they are published or not. The documents may come from teaching and research institutions in France or abroad, or from public or private research centers.
L'archive ouverte pluridisciplinaire HAL, est destinée au dépôt et à la diffusion de documents scientifiques de niveau recherche, publiés ou non, émanant des établissements d'enseignement et de recherche français ou étrangers, des laboratoires publics ou privés. 


\section{É G Y P T E C H R É T I E N N E E T A R A B E}

\section{La première pétition en copte : révision de SB Kopt. IV 1709}

Les procédures judiciaires de l'Égypte protobyzantine ont beau être mal connues et attendre qu'on leur consacre un travail systématique prenant en compte l'accroissement de la documentation éditée depuis les études pionnières, fondées sur des papyrus déjà anciens $\left({ }^{1}\right)$, il est un fait sur lequel on s'accorde, c'est qu'elles nécessitaient le recours exclusif à la langue grecque. La pétition, pièce maîtresse du dispositif judiciaire, par laquelle le plaignant portait à la connaissance des autorités un préjudice qu'il aurait subi, soit en en demandant l'immédiate réparation, soit en sollicitant l'ouverture d'un procès, devait être rédigée en grec, langue de l'État, au nom d'un principe qui remonte à la naissance du royaume lagide et auquel l'administration romaine d'Égypte ne dérogera pas.

Cette situation de monolinguisme ne pouvait manquer de créer des difficultés et des frustrations dans une population qui, dans sa majorité, ne maîtrisait pas le grec et qui, avec l'invention et la montée en puissance du copte, pouvait espérer que son système judiciaire fît une place croissante à la langue égyptienne. Or, durant les $\mathrm{IV}^{\mathrm{e}}-\mathrm{VII}^{\mathrm{e}}$ siècles, les pétitions dont nous disposons sont toutes en grec. Ce qui pourrait passer pour une forme d'intolérance linguistique de la part de l'administration a été, estime-t-on, à l'origine du développement de procédures extra-judiciaires comme l'arbitrage, qui devient de plus en plus fréquent à partir

(1) Notamment les P. Lips. I 33, P. Oxy. I 67 et P. Oxy. XVI 1876-1882, dont l'édition bouleversa bien des idées reçues et suscita de nombreux travaux. Voir principalement P. CollinET, $L a$ procédure par libelle (Paris, 1932). L. MiTTEIs dans ses Grundzüge und Chrestomathie (Leipzig, 1912), qui reste encore un ouvrage de référence pour beaucoup de papyrologues et d'historiens, ne consacre que quelques lignes à la période byzantine (et encore s'agit-il surtout du IV siècle ; cf. aussi L. MitTEIS, «Zur Lehre von den Libellen und der Prozesseinleitung nach den Papyri der früheren Kaiserzeit », Berichte über die Verhandlungen der königlich sächsischen Gesellschaft der Wissenschaften zu Leipzig, Phil.-hist. Kl. 62 [1910], pp. 61-126, en part. pp. 106-121). Il n'existe toujours pas, pour la période byzantine, d'équivalent aux ouvrages de H.J. WolfF, Das Justizwesen der Ptolemäer (Munich, 1970²) pour l'époque ptolémaïque, et de G. Foti TALAmanca, Ricerche sul processo nell'Egitto greco-romano. I. L'organizzazione del 'conventus' del 'praefectus Aegypti' (Milan, 1974) ; II. L'introduzione del giudizio. 1 (Milan, 1979), ou B. Kelly, Petitions, Litigation, and Social Control in Roman Egypt (Oxford, 2011) pour l'époque romaine.

Chronique d'Égypte XCIII (2018), fasc. 185 - doi: 10.1484/J.CDE.5.116103 
du IV ${ }^{\mathrm{e}}$ siècle $\left(^{2}\right)$ : le règlement à l'amiable pouvait en effet constituer, pour la partie non-hellénophone de la population, un moyen de résoudre ses litiges sans avoir à passer par la voie ordinaire, laquelle impliquait, à chaque étape (pétition, audience), l'usage obligatoire du grec.

Pourtant, le monolinguisme de l'Égypte byzantine dans le domaine du droit commence à se fissurer à partir de la seconde moitié du $\mathrm{VI}^{\mathrm{e}}$ siècle avec l'apparition de textes juridiques en copte $\left(^{3}\right)$. On s'attendrait à ce que cette évolution se soit accompagnée d'une plus grande tolérance pour l'emploi du copte dans le domaine judiciaire, là où précisément les textes juridiques, justifiant les droits des plaignants, donnaient lieu à des procès. Le recours au copte pour pétitionner auprès des autorités aurait dû être une des conséquences logiques de ce changement - a fortiori après que l'Égypte eut coupé ses liens avec Byzance à la suite de la conquête arabo-musulmane. Pourtant, la documentation papyrologique passe pour n'avoir livré aucun spécimen de pétition stricto sensu en copte.

En fait, un contre-exemple a bien été publié il y a quatre-vingts ans et son éditeur en avait entrevu l'importance pour l'histoire du copte, mais, présenté comme une lettre, il n'a pas retenu toute l'attention qu'il méritait. Par ailleurs, certaines parties essentielles à sa compréhension avaient résisté au déchiffrement. Ce papyrus nécessite donc une réédition qui en montrera tout l'intérêt pour l'histoire des institutions judiciaires de l'Égypte byzantine.

Le document appartient à la collection de Vienne (P. Vindob. Inv. K 950) et a été édité en 1938 par Walter C. Till. Le texte a été reproduit ensuite par le même Till dans la chrestomathie de sa grammaire parue en 1961 et finalement repris en 2012 dans SB Kopt. IV 1709, avec des corrections de M. Hasitzka $\left({ }^{4}\right)$.

(2) Sur l'arbitrage, voir A. STEINWENTER, « Das byzantinische Dialysis-Formular », Studi in memoria di Aldo Albertoni, I (Padoue, 1932), pp. 75-94; J. MoDRZEJEWSKI, « Private Arbitration in the Law of Greco-Roman Egypt », JJP 6 (1952), pp. 239-256 ; T. GAGOS \& P. VAN MinNEN, Settling a Dispute. Toward a Legal Anthropology of Late Antique Egypt (Ann Arbor, 1994) (liste des textes à partir de la fin du III ${ }^{\mathrm{e}}$ siècle de notre ère, pp. 121-127, à compléter par C. KREUZSALER [cf. ci-après], p. 23, n. 27) ; J. URBANIK, « Compromesso o processo? Alternativa risoluzione dei conflitti e tutela dei diritti nella prassi della tarda antichità », Symposion 2005. Vorträge zur griechischen und hellenistischen Rechtsgeschichte (Vienne, 2007), pp. 377-400 ; C. KreuzSAler, "Die Beurkundung aussergerichtlicher Streitbelegung in den ägyptischen Papyri », Quellen zur byzantinischen Rechtspraxis. Aspekte der Textüberlieferung, Paläographie und Diplomatik (Vienne, 2010), pp. 17-26 et Kelly, Petitions [n. 1], chap. 7, pp. 244-286.

(3) Voir récemment J.-L. FourNET, «Sur les premiers documents juridiques coptes », Études coptes XI. Troisième journée d'étude (Marseille, 7-9 juin 2007) (Paris, 2010), pp. 125-137; J.-L. Fournet, H. FörSTER \& T.S. RichteR, «Une misthôsis copte d'Aphrodité (P.Lond. inv. 2849) : le plus ancien acte notarié copte? », ArchPF 58 (2012), pp. 344-359 ; J.-L. FouRnET, "Sur les premiers documents juridiques coptes (2) : Les archives de Phoibammôn et de Kollouthos », Études coptes XIV. Seizième journée d'études coptes (Genève, 19-21 juin 2013) (Paris, 2016), pp. 115-141 et Egyptian versus Greek in Late Antique Egypt: The Quest of Coptic for an Official Status, sous presse à la Princeton University Press, chap. III.

(4) W.C. TILL, "Eine koptische Alimentenforderung », BSAC 4 (1938), pp. 71-78 (= Till ${ }^{1}$ ) et Koptische Grammatik (Leipzig, 1961²), pp. 313-314, où le document est repris sous le titre 
Deux traductions, en allemand et en anglais, en ont par ailleurs été fournies en 1995 et $1998\left(^{5}\right)$.

L'image en ligne de ce papyrus permet d'en donner une édition corrigée et complétée de l'endossement et d'une ligne dans la marge supérieure du recto que l'éditeur n'avait pu lire. Cette dernière a d'ailleurs le mérite de nous apprendre que ce papyrus, jusqu'ici de provenance inconnue, vient d'Edfou. Quoiqu'ils n'aient pas de rapport direct avec le reste du document, nous ajoutons en annexe une édition des comptes, inédits, qui couvrent une partie du verso.

P. Vindob. Inv. K 950 (= SB Kopt. IV 1709) $8 \times 34,5 \mathrm{~cm}($ FigG. 3-4)

Apollinopolis Anô (Edfou) $1^{\text {re }}$ moitié du VII ${ }^{\mathrm{e}}$ siècle

Coupon de papyrus de forme rectangulaire. La marge inférieure est perdue. Plusieurs blocs de texte sont disposés sur le recto et le verso (voir figg. 1-2). Au recto $(\downarrow)$, une première main a écrit transversa charta un texte copte dont les 9 lignes occupent la majeure partie du feuillet (A). Dans les marges supérieure et inférieure, des séquences ont été ensuite notées tête-bêche par rapport à A. Dans le bas du feuillet, deux lignes sont visibles (B) : il s'agit de la fin d'un texte copte, dont il manque au moins une ligne, peut-être davantage. Ces deux lignes sont écrites par deux nouvelles mains $\left(\mathrm{m}^{2}\right.$ et $\left.\mathrm{m}^{3}\right)$. Au-dessus du texte A, toujours tête-bêche, une ligne a été écrite en grec, peut-être par une ou plusieurs nouvelles mains, voire par la $1^{\text {re }}$ main mais dans des styles différents. $\mathrm{Au}$ verso $(\rightarrow)$, environ au centre du feuillet, dans le même sens que A, se trouvent trois lignes, écrites de la même main que le début de la séquence $\mathrm{C}$, c'est-à-dire peut-être la $1^{\text {re }}$ main. En effet, si l'interprétation que nous développons ci-après est correcte, la $1^{\text {re }}$ main aurait eu plusieurs styles, le premier, $\mathrm{m}^{1}-\mathrm{s}^{1}$, pour le texte principal du recto (A), et un ou deux autres, $\mathrm{m}^{1}-\mathrm{s}^{2}$ et $\mathrm{m}^{1}-\mathrm{s}^{3}$, pour l'endossement du verso (D) et la ligne de C, qui joue le rôle de prescrit $\left({ }^{6}\right)$. Enfin, une nouvelle et dernière main a réutilisé le document pour noter trois comptes dans les espaces vierges du verso (E1-E3, édités en annexe).

«Ansuchen um Rechtshilfe » $\left(=\right.$ Till $\left.^{2}\right)$. Nous remercions vivement la Papyrussammlung de Vienne et en particulier Mme Claudia Kreuszaler de nous avoir autorisés à reproduire l'image du papyrus.

(5) La traduction allemande, avec la photo du papyrus, est parue dans H. BusCHHAUSEN, U. Horak \& H. Harrauer (edd.), Der Lebenskreis der Kopten. Dokumente, Textilien, Funde, Ausgrabungen. Katalog zur Ausstellung im Prunksaal der Österreichischen Nationalbibliothek. 23. Mai bis 26. Oktober 1995 (Vienne, 1995), pp. 10-11, n 10 (« Keine Alimente für die kranke Frau »). La traduction anglaise est fournie dans J. RowLANDSON (ed.), Women and Society in Greek and Roman Egypt. A Sourcebook (Cambridge, 1998), p. 216, n ${ }^{\circ} 160$ sous le titre "Complaints over unpaid alimony ». Le papyrus a fait l'objet d'une présentation et d'une discussion à la conférence EPHE d'Alain Delattre en 2017. Enfin, très récemment, il a été brièvement rendu compte du document sur le blog « Papyrus Stories. Ancient Lives from the Past» (< https://papyrus-stories. com/2018/05/12/an-abandoned-wife-and-unpaid-alimony $>$ ).

(6) Pour « $\mathrm{s} »$ désignant le style d'écriture indépendamment de la main (« $\mathrm{m} »)$, cf. P. Worp, p. 248 , n. 28 . 
On notera enfin que le papyrus présente des traces de plis verticaux et horizontaux sur sa surface. De même, on remarque au verso, entre les plis, une zone plus foncée qui a dû être exposée plus longtemps aux éléments. Ils permettent de reconstituer la manière dont le papyrus avait été plié lors de sa première utilisation. Le papyrus a été plié une première fois selon un axe vertical, au centre du coupon, ensuite il a été plié deux fois selon l'axe horizontal, de manière à replier le tiers supérieur et le tiers inférieur sous le tiers médian. Le petit coupon ainsi constitué a été plié une dernière fois en deux selon l'axe vertical. Sur cet espace de $3 \times 8,5 \mathrm{~cm}$ qui correspond au douzième de la surface du coupon, le scribe a noté l'endossement (D).

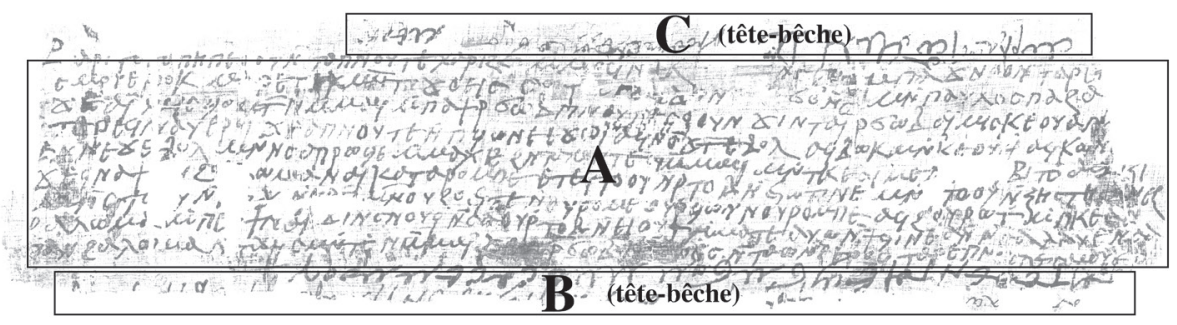

FIG. 1. - Disposition des textes au recto de P. Vindob. Inv. K 950 (= SB Kopt. IV 1709)

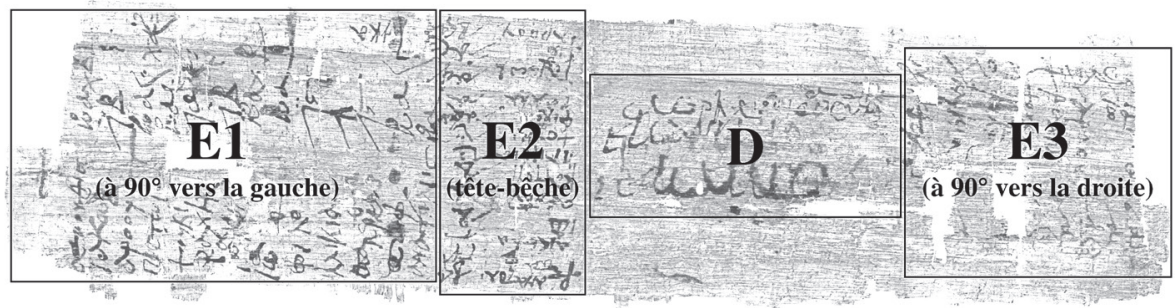

FIG. 2. - Disposition des textes au verso de P. Vindob. Inv. K 950 (= SB Kopt. IV 1709) 


\section{Recto}

$\mathrm{A} \downarrow$

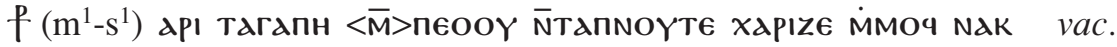

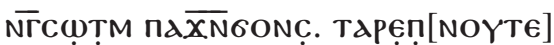

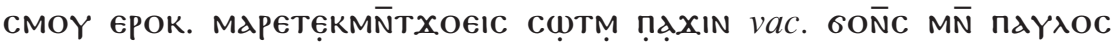
пג2d!. [0-4]

$x \in$ גїMIC KeOYa NM[MAY.]

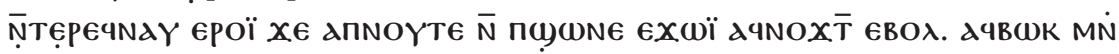

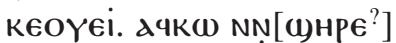

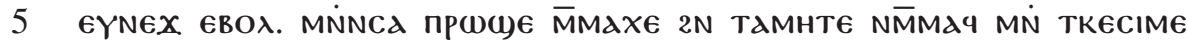
NṬ[a]

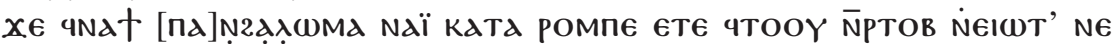
MN qTOOY ŇrHCTHC ṆNG?

$M \bar{N}$ qTọ[O]Y N̄ NOYPOMПE. Ач2OYPWT' M̈RKEAN-

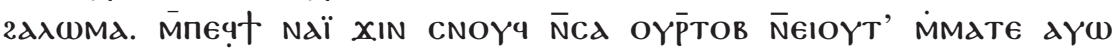
$\bar{N}$ TUINE $d \bar{N} \bar{N} C d \lambda d \gamma \in \bar{N} C d$

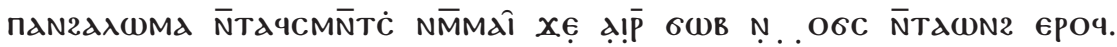
TAPEחNOYTE CMOY EPOK.

En bas du feuillet, écrit tête-bêche :

$\mathrm{B} \downarrow$

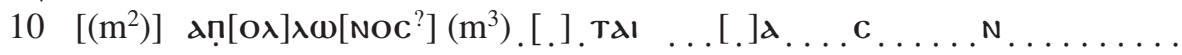
[.].[..].[ $[ \pm$ 4]

([ ]) Tal Te eE NTAie() gMGOM mMecace mMOY. †

En haut du feuillet, écrit tête-bêche :

$\mathrm{C} \downarrow$

$\left(\mathrm{m}^{1}-\mathrm{s}^{2}\right)$ A ̉̉

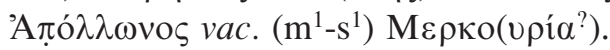

VERSO

Au milieu du feuillet (dans le même sens que A) :

$\mathrm{D} \rightarrow$

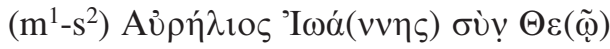

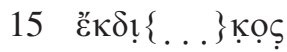

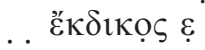




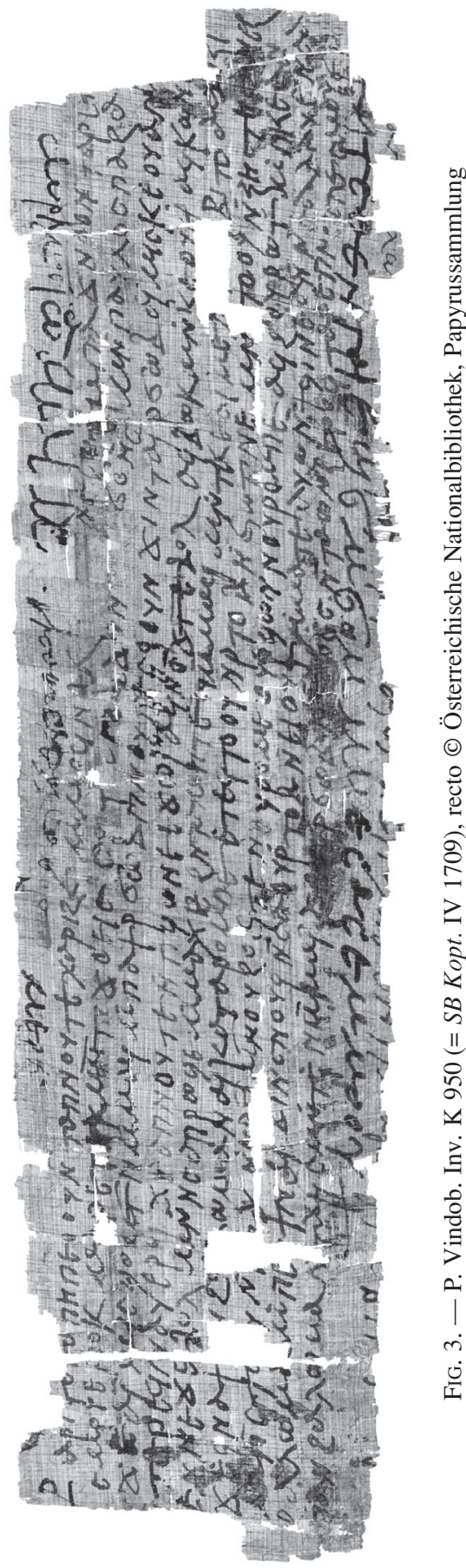




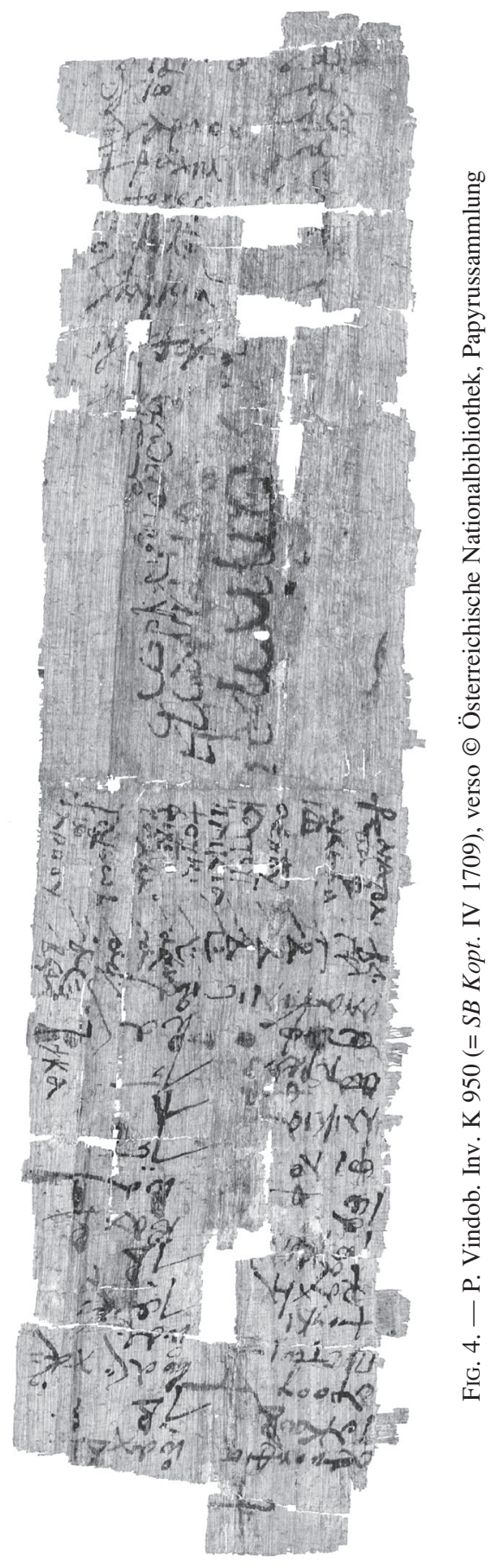




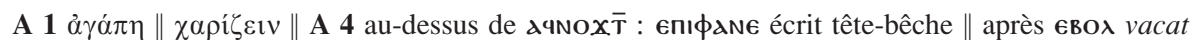
d'une lettre $\|$ kєoүєi : le k est curieusement surmonté d'un signe ressemblant à une apostrophe $\|$

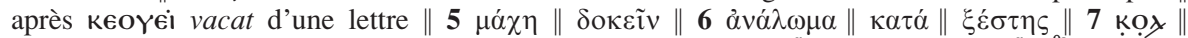

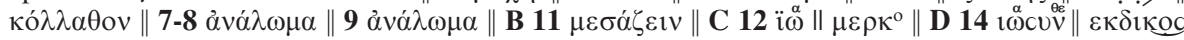

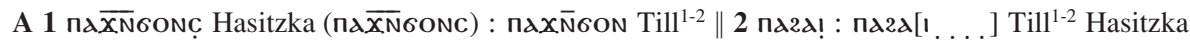

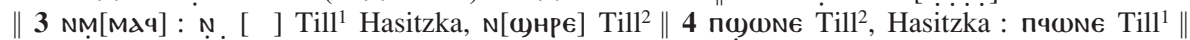

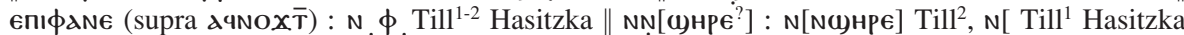

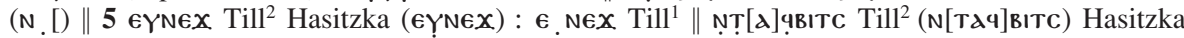

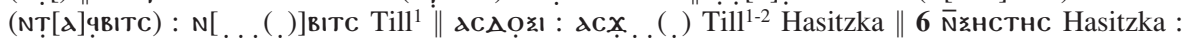

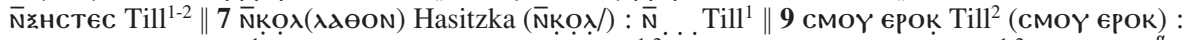
смоүє1 єр[ок] Till ${ }^{1}$ Hasitzka $\|$ B 10 non leg. Till ${ }^{1-2}$ Hasitzka $\|$ C non leg. Till ${ }^{1-2}$ (praeter $\ddot{i} \omega$ ), A $\rho \eta \overline{\lambda \operatorname{lo} \varsigma} \mathrm{I} \omega \alpha \ldots . . . v \eta \varsigma \alpha \pi \alpha \ldots$. . . . . . ME . . Hasitzka II D non leg. Till ${ }^{1-2}$ (praeter $\left.\ddot{i} \stackrel{\alpha}{\omega}\right)$ Hasitzka $\| \mathbf{E}$ non ed. Till ${ }^{1-2}$ Hasitzka

A « Aie la bonté, <par> la gloire dont Dieu t'a gratifié, d'écouter l'injustice dont je suis victime, et que Dieu te bénisse! Puisse Ta Seigneurie écouter l'injustice (que j'endure) avec Paul, mon mari. J'ai eu trois enfants de lui avant de tomber souffrante et Dieu sait qu'à partir du moment où j'ai été souffrante, j'ai eu un autre enfant avec lui. Lorsqu'il vit que Dieu m'avait apporté la maladie, il m'a chassée. Il est parti avec une autre. Il a laissé les enfants?, abandonnés. À la suite de la copieuse dispute entre moi et lui ainsi que l'autre femme qu'il avait prise, il a été décidé qu'il me donnerait une pension chaque année, d'un montant de quatre artabes d'orge, de quatre setiers d'huile, de quatre kollatha de vin, d'un manteau pour un an et d'une couverture pour un an. $<\mathrm{Or}$, $>$ il m'a privée de la pension même : il ne m'a rien donné depuis l'an dernier, si ce n'est seulement une artabe d'orge. Et je ne demande rien d'autre que la pension qu'il a fixée avec moi parce que je suis souffrante, ... que je puisse en vivre. Que Dieu te bénisse ».

B «... Apollônos? ... voilà comment j'ai pu trancher l'affaire entre eux ».

C « $<$ À $>$ Aurelius Iôannês, defensor d'Apollônos Anô, Merkouria? ».

D « $<$ À $>$ Aurelius Iôannês, par la grâce de Dieu defensor... defensor ».

1-2 Le vacat qui interrompt les deux lignes (plus large à la première) pourrait être dû à un défaut dans le papyrus, que la rédactrice aura pris soin d'éviter.

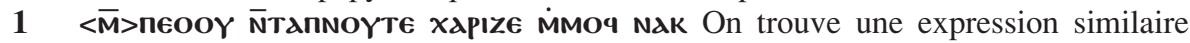
dans SB Kopt. III 1274, 6 : M̄neooy ntannoyte tadq $\bar{N}$. . [, « par la gloire que Dieu a donnée à ....».

NīCoTM na $\overline{\mathbf{X N}}$ GONC L'expression trouve un bon parallèle dans $O$. Crum Ad. 65,

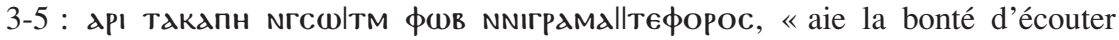
l'affaire de ces personnes qui t'apportent la lettre ». On peut se demander s'il ne faut pas interpréter de la même manière $O$. Crum 187, 10 : ] H NrCWTM naxIN . N, peut-être à comprendre NrCWTM naxINGONC, "écoute l'injustice que je subis » (l'espace semble manquer pour restituer $\lambda$ pı TגГגח]ب̣ en tête de la séquence). na $\overline{\mathbf{X N}}$ GONc Le $\mathrm{c}$ est minuscule et collé contre le $\mathrm{N}$. 
3 XıNTdī dans la séquence $+\overline{\mathrm{P}} \sigma \omega \mathrm{B}$ qui précède (où elle se réduit quasiment à un point comme c'est parfois le cas ailleurs, ce qui explique que Till ne l'ait pas remarquée).

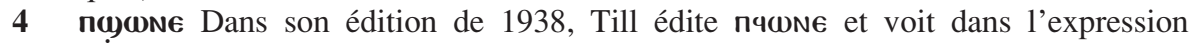

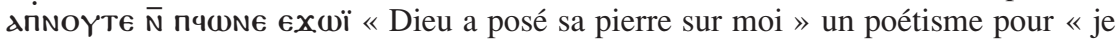
suis tombé malade » (repris dans BuschHausen, HoraK \& Harrauer [edd.], Der Lebenskreis der Kopten [n. 5], p. 10). En fait, les $\boldsymbol{\omega}$ ) de ce texte ont une seconde « cuvette » tellement atrophiée que la lettre peut ressembler à un 4 .

$\mathbf{N N}\left[\mathbf{\omega} \mathbf{H P G}^{?}\right]$ La restitution est un peu longue pour la lacune, mais elle est incontournable. La rédactrice a dû serrer les lettres en fin de ligne.

5 aCAOå Le verbe montre que les deux parties se sont mises d'accord, vraisemblablement dans le cadre d'une procédure d'arbitrage. On comparera la formulation, par exemple, à celle du contrat de mariage $S B$ Kopt. II 933, $3:$ ac $\Delta O z_{2}$ OYN $2 \bar{N}$

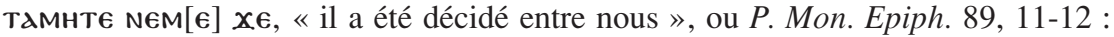

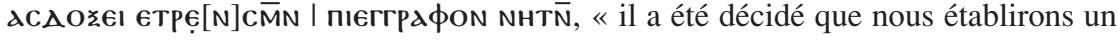
document pour vous ».

6 [nd]м̣2ạ.

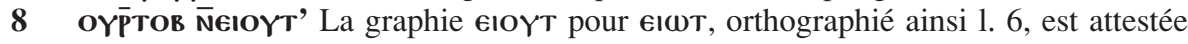
quelques fois : une occurrence littéraire est mentionnée dans Crum, Dict., 87a ; pour le domaine documentaire, le terme apparaît sous cette forme dans $O$. Brit. Mus. Copt. I, pl. LXI, 5, 7-8. La confusion entre oy et $\omega$ est fréquente surtout dans la région thébaine (cf. P. KAHLE, Bala'izah. Coptic Texts from Deir el-Bala'izah in Upper Egypt [Londres, 1954], vol. I, p. 90).

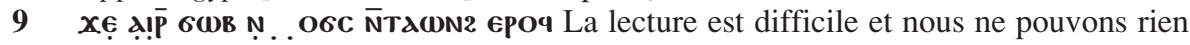
proposer de plus que Till : "Was in Zeile 9 nach $x \in$ dı $6 \omega$ B denn ich bin krank geworden steht, kann ich nicht lesen. Es sind einige Buchstaben gänzlich verschmiert. Der Buchstabe vor $\sigma$ kann auch $\omega$ sein. Der Sinn der Stelle ist jedenfalls der : da ich krank bin und mir infolge dessen kein anderes Einkommen verschaffen kann, habe ich nur die Alimentation zum leben und bin daher gezwungen, auf ihrer völlstandigen Ausfolgung zu bestehen. » (TILL, « Eine koptische Alimentenforderung » [n. 4], pp. 77-78).

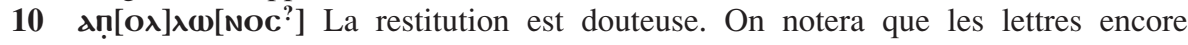
visibles sont tracées au moyen d'un calame plus fin et dans un plus petit module que celles du reste de la ligne et de la suivante.

11 mecace Le mot grec $\mu \varepsilon \sigma a ́ \zeta \varepsilon ı v$ se retrouve en copte dans des partages de succession, où il a le sens de « diviser qqch (MMO") entre $(\epsilon \times \omega=)$ (Förster, $W B, s . v . \mu \varepsilon \sigma \alpha ́ \zeta \omega$, p. 516), d'où la traduction de Till (« Auf diese Weise war ich imstande, sie (pl.) zu

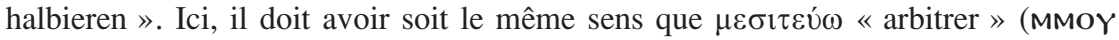
renvoyant aux deux parties), soit le sens de "diviser », d'où " trancher », syno-

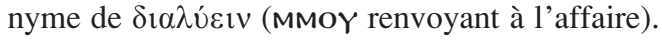

14 бòy $\Theta \varepsilon(\tilde{\varphi})$ L'abréviation $\Theta \varepsilon(\tilde{\omega})$ au lieu de $\Theta(\varepsilon \tilde{\omega})$ est inhabituelle.

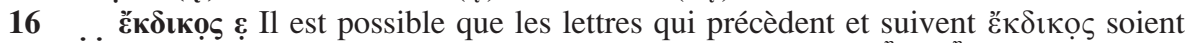
les restes d'un texte sous-jacent. Au début, on pourrait lire $\zeta^{\eta} \tau$ ou $\stackrel{n}{\pi}=(\kappa \alpha i) \tau \eta()$ ou $\pi \eta($ ). On lirait peut-être plus naturellement $\varepsilon \kappa \delta ı \kappa v c$ (avec un petit $v$ en hauteur comme cela arrive avec certaines voyelles dans l'écriture des endossements), mais la faute serait surprenante, même en tenant compte du bas niveau de grec de la personne qui écrit. Aussi nous paraît-il plus économique de considérer que le o a été écrit en «monogramme » avec la lettre qui précède et celle qui suit. 
Le texte du verso (A) est une plainte adressée par une dame qui réclame une pension à son mari après que celui-ci a quitté le domicile conjugal. Dans un premier temps, le litige entre les époux a fait l'objet d'un arbitrage où il fut décidé (1. 5 : גCAỌ̌ı) que le mari verserait une pension à sa femme. Mais celuici ne tint pas ses engagements et l'épouse décida d'en appeler à la justice du destinataire de cette plainte.

Du point de vue du contenu, cette plainte ressemble donc fort à une pétition au sens strict du terme. On doit cependant avoir à l'esprit qu'à cette époque, les lettres empruntent régulièrement à la rhétorique des pétitions, surtout quand elles transmettent une requête. Certaines de ces demandes épistolaires pouvaient à ce point mimer la forme des pétitions qu'on a du mal à les distinguer des pétitions stricto sensu, si ce n'est quand la qualité de leur destinataire échappe à la catégorie des gouverneurs ou des magistrats municipaux $\left(^{7}\right)$. Aussi, pour bien interpréter notre plainte, est-il nécessaire de soulever la question de son destinataire. L'absence de prescrit - il ne manque aucune ligne au début du texte - a favorisé diverses hypothèses quant à sa qualité. La plus récente consistait à y voir « a religious official, a local priest or bishop » $\left({ }^{8}\right)$. Or, dans la mesure où elle n'était pas une moniale, la plaignante aurait échappé à la juridiction de l'évêque, qui, dans cette affaire, n'aurait eu qu'un rôle d'arbitre ou d'intermédiaire auprès des autorités civiles. Autrement dit, cette plainte ne présenterait pas les caractères d'un libelle soumis aux autorités judiciaires, mais bien d'une supplique épistolaire ou demande d'intercession. On a d'autres exemples de ce type de prières adressées à des évêques (par exemple, pour un document écrit par une femme, SB Kopt. I 295). Leur caractère informel pourrait d'ailleurs expliquer le recours au copte.

Walter C. Till était pourtant d'avis que cette plainte, de forme épistolaire comme il le précise, avait plutôt pour destinataire un fonctionnaire public (un fonctionnaire villageois), sinon, une personne influente $\left(^{9}\right)$. Les éditeurs du catalogue Der Lebenskreis der Kopten penchaient aussi pour « eine Persönlichkeit des öffentlichen Lebens » $\left({ }^{10}\right)$.

(7) J. GASCOU, «Les pétitions privées », La pétition à Byzance (Paris, 2004), pp. 93-103 (reproduit dans J. Gascou, Fiscalité et société en Égypte byzantine [Paris, 2008], pp. 125-213, IX).

(8) Rowlandson (ed.), Women and Society [n. 5], p. 216.

(9) TILL, «Eine koptische Alimentenforderung » [n. 4], p. 73 : « Es ist also anzunehmen, dass der Adressat eine öffentliche Funktion bekleidet, die ihn dazu berufen erscheinen lässt, in solchen Fällen zum Rechten zu sehen [...]. Wie in so vielen anderen Fällen wird auch hier der Streitfall wohl zunächst einem Dorffunktionär oder sonst einem einflussreichen Mann vorgetragen und dieser wird nun kraft seiner Autorität versuchen, die Sache in Ordnung zu bringen ».

(10) Buschinusen, HoraK \& Harrauer (edd.), Der Lebenskreis der Kopten [n. 5], p. 10. 
Les parties que Till n'a pu déchiffrer donnent en fait la solution et confirment son intuition. Ce qui s'apparente, à la fois par la position (sur le verso) et par l'écriture (un style d'écriture droite, de gros module et privilégiant la verticalité des hastes), à un endossement de lettre ou de pétition est susceptible de nous

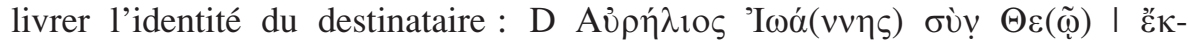
$\delta \mathfrak{1}\{\ldots\}$. . de la mention d'un defensor civitatis avec une plainte ne peut être contingente : ce fonctionnaire, créé par Constantin, avait comme vocation première de défendre la plèbe «contre les abus des puissants » (contra potentium iniurias) $\left({ }^{11}\right)$, mission que réaffirma Justinien en lui octroyant plus de pouvoir face aux gouverneurs, en lui conférant une juridiction dans des causes criminelles mineures et en obligeant les plaignants, dans une affaire dont la valeur litigieuse ne dépasse pas 300 solidi, à s'adresser à lui plutôt qu'au gouverneur $\left({ }^{12}\right)$. L'affaire de notre plaignante, qui impliquait de petites sommes, était donc tout à fait du ressort de ce fonctionnaire. Sa pétition aurait donc été transmise à un defensor civitatis.

Cette explication se heurte toutefois à la grammaire : si le defensor était le destinataire de cette plainte, l'adresse qui mentionne son nom devrait être au datif et non au nominatif. Nous pensons pourtant qu'il pourrait s'agir d'une erreur de la rédactrice, qui, peu à l'aise en grec (d'où le recours au copte pour la plainte elle-même), se serait trompée dans le choix du cas. Son manque de maîtrise en grec se manifeste par une écriture maladroite qui tente de reproduire le style des endossements épistolaires - sans grand succès d'ailleurs - et par une hésitation sur la forme du nom de fonction du destinataire : estropié une première fois $(\tilde{\varepsilon} \kappa \delta ! \ldots \kappa ̣ \varsigma)$, le mot est réitéré à la ligne suivante mais avec une désinence peu claire.

C'est peut-être la raison pour laquelle notre plaignante a réécrit cette adresse dans la marge supérieure du recto, tête-bêche par rapport au texte de la plainte :

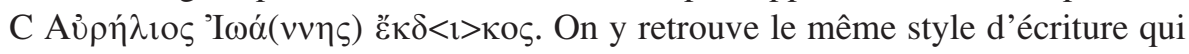
montre qu'il s'agit bien d'une adresse, quoique sur le recto $\left({ }^{13}\right)$. L'erreur de cas est répétée, mais la désinence du nom de la fonction est cette fois-ci écrite avec plus de clarté - à ceci près qu'il manque une haste verticale dans la séquence qui la précède (comme si le $\mathrm{l}$ ou un élément du $\kappa$ avait été omis). Le ressort géographique du defensor ( $\tau \tilde{\eta} \varsigma$ ợ

(11) L'expression est empruntée à un texte plus récent, Cod. Théod. I, 29, 1 (364 ou 368) concernant l'Illyricum. Pour la date, débattue, cf. R.M. FraKes, Contra Potentium Iniurias: The Defensor Civitatis and Late Roman Justice (Munich, 2001), pp. 94-104, qui opte pour 364.

(12) Nov. XV, 3. Pour le defensor, voir en dernier lieu FraKES, Contra Potentium Iniurias [n. 11].

(13) Sa position (tête-bêche par rapport au texte de la pétition) montre bien qu'il ne s'agit pas d'un prescrit, même si elle finit par en remplir le rôle. 
autre main, à moins que ce ne soit par la plaignante elle-même, qui abandonne le style des endossements épistolaires pour celui de la protominuscule grecque.

Enfin, $\mathrm{C}$ se termine par la mention $\mathrm{M} \varepsilon \rho \kappa \mathrm{(} \mathrm{),} \mathrm{écrite} \mathrm{de} \mathrm{la} \mathrm{même} \mathrm{main} \mathrm{et} \mathrm{dans}$ le même style que le reste de la plainte. Si cette ligne joue le rôle d'une adresse, normalement constituée de l'inscriptio (nom du destinataire) et de l'intitulatio

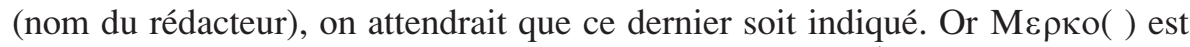

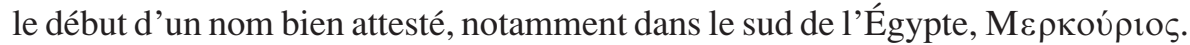
S'il faut bien y voir le nom de la plaignante, on doit alors résoudre l'abréviation du nom avec une désinence féminine : Мєрко( $\rho i ́ \alpha)$. Cette forme existe bien $\left({ }^{14}\right)$, mais il peut paraître étonnant qu'on ait abrégé un nom dont la forme masculine est bien plus courante. Il est vrai que, dans la procédure de délivrance d'une pétition, le plaignant soumet sa plainte en personne et peut exposer son cas oralement à la demande du magistrat, ce qui permet de lever les ambiguïtés que peut contenir le document remis. N'oublions pas non plus que notre pétitionnaire est loin de maîtriser les codes écrits du grec $\left({ }^{15}\right)$. Le fait qu'elle utilise une adresse de type épistolaire (où l'intitulatio est au nominatif) et qu'elle ait rédigé son texte transversa charta $\left({ }^{16}\right)$ en sont des indices supplémentaires - encore que non décisifs dans un contexte où le copte est avant tout la langue des lettres.

On peut se demander pourquoi notre plaignante - appelons-la désormais Merkouria - utilise le grec pour cet « endossement » alors qu'elle connaît manifestement mal cette langue et qu'elle a recours au copte pour la plainte ellemême. La raison en est probablement l'influence que la diplomatique grecque a exercée sur les documents coptes : pendant longtemps, les rédacteurs de lettres ou de documents juridiques en copte ont continué à utiliser le grec pour les endossements du fait du statut institutionnel dont jouissait cette langue $\left({ }^{17}\right)$.

(14) P. Lond. Copt. I 1111, 19 (Hermoupolis, sans date).

(15) On notera cependant qu'elle se conforme à l'opposition de styles graphiques que l'on observe, dans les endossements épistolaires ou les prescrits de pétitions grecques, entre l'inscriptio (écriture plus stylisée et solennelle) et l'intitulatio (écriture simple). Cf. J.-L. FourNET, « Anatomie d'un genre en mutation : la pétition de l'Antiquité tardive », Pap. Congr. XXVIII (sous presse).

(16) Cf. J.-L. Fournet, « Disposition et réalisation graphique des lettres et des pétitions protobyzantines : pour une paléographie "signifiante" des papyrus documentaires », Proceedings of the 24th International Congress of Papyrology. Helsinki, 1-7 August, 2004 (Helsinki, 2007), pp. 353 367.

(17) J.-L. FourNET, « Les documents bilingues gréco-coptes dans l’Égypte byzantine : essai de typologie », Written Sources about Africa and their Study / Le fonti scritte sull'Africa e i loro studi (Milan, 2018), pp. 59-83 : l'opposition texte du recto (copte) $\neq$ endossement (grec) est une de ces alternances « extrasectionnelles » qui entrent dans la catégorie des alternances extraphrastiques (extra-sentential code switching), dans lesquelles le grec est utilisé pour tout ce qui relève de l'institutionnel (datation impériale, invocation divine, nom de l'autorité à qui est adressé un document, date, sommes ou quantités, complétion notariale, etc.). 
Toujours est-il qu'ayant retrouvé son adresse avec son inscriptio et, pensonsnous, son intitulatio, notre document est bien doté des éléments dont l'absence semblait constituer aux yeux du premier éditeur la preuve qu'on avait affaire à un brouillon $\left({ }^{18}\right)$. Certes la présentation du texte et son écriture sont peu soignées, avec, on l'a vu, la réécriture de certaines séquences qui peuvent aller dans le sens d'une ébauche, mais un élément achève de nous convaincre du contraire : il s'agit des lignes écrites dans la marge inférieure du recto du feuillet, là encore tête-bêche (B). Seule la dernière est encore visible et elle s'apparente à la conclusion d'une sorte de rescrit ou, tout du moins, d'apostille officielle : Tal тє өє NTAiE() 6M6OM MMECגCE MMOY «voilà comment j'ai pu trancher l'affaire entre eux ». Il peut s'agir soit d'une apostille par laquelle le defensor explique comment il a procédé à la résolution du litige, soit de la réponse d'un autre fonctionnaire auquel le defensor a renvoyé l'instruction de l'affaire. Quoi qu'il en soit, il s'agit bien d'un texte appartenant à une phase ultérieure à la présentation de la pétition. On pourrait objecter que son écriture est très similaire à celle du texte de la plainte $\left({ }^{19}\right)$. Il n'est pas impossible que ce soit alors la plaignante qui ait recopié sur sa pétition la décision que lui aura faite le defensor. Mais on peut aussi défendre l'idée qu'il s'agit d'une autre main : le $\gamma$ final est très différent de ceux du texte A; le 6 n'a pas la fin recourbée qui s'observe en A; le $\omega$ ) a sa seconde « cuvette » aussi large que la première contrairement à ceux de $\mathrm{A}\left({ }^{20}\right)$.

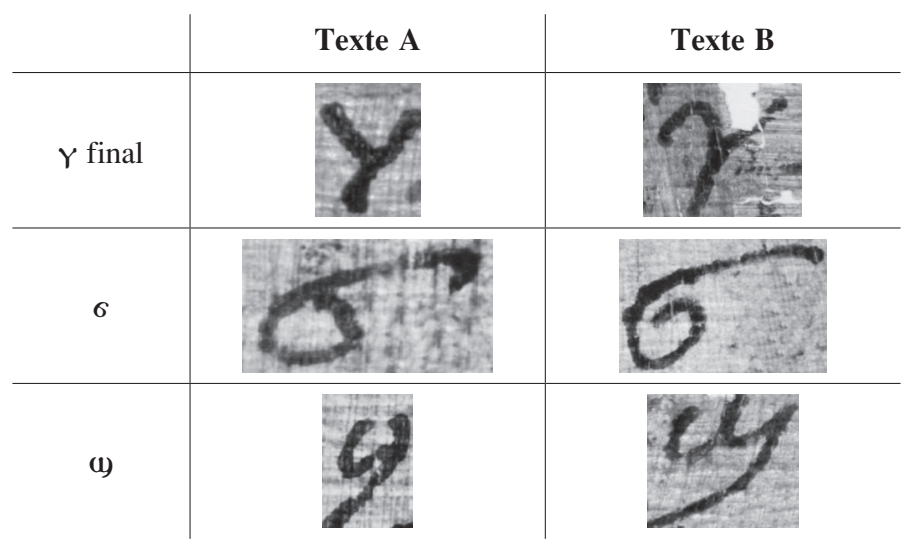

(18) TILL, «Eine koptische Alimentenforderung » [n. 4], p. 73 : « Ich wollte nur auf die Möglichkeit hinweisen, dass es sich hier um ein Konzept handeln könnte, was einige Fahrlässigkeiten in der Stilisierung begreiflich erscheinen lassen würde, vor allem das Fehlen der Angabe, von wem die Eingabe gemacht wurde ».

(19) TiLL, «Eine koptische Alimentenforderung » [n. 4], p. 71, retient qu'elle est identique à celle de l'adresse $(\mathrm{C}$ et $\mathrm{D})$, alors qu'il nous semble qu'on a affaire à deux styles d'écriture différents.

(20) Au point que Till a confondu le a) et le ч dans un mot de la 1. 4 (cf. note du texte $a d l o c$.). 
Aussi est-il plus économique de conclure que le defensor a apposé (lui-même ou par la main d'un secrétaire) sa décision sur le même feuillet que celui de la pétition. Nous n'avons pas, à notre connaissance, d'autres exemples de réponse

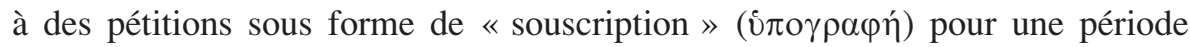
aussi récente - les derniers datent du $\mathrm{IV}^{\mathrm{e}}$ siècle $\left({ }^{21}\right)$-, mais le caractère sans nul doute plus informel de la justice rendue par ce juge des «petits » qu'était le defensor peut expliquer ce qui nous apparaît aujourd'hui comme une irrégularité, en même temps d'ailleurs que l'allure peu soignée du document - aussi bien dans la partie due à la plaignante qu'à celle émanant du fonctionnaire ou de son bureau. Il est vrai aussi que nous conservons peu de documents nous renseignant sur la façon précise dont le defensor procédait dans l'instruction des affaires qui lui étaient soumises.

Nous venons de parler de «période récente » : aussi est-il temps d'aborder le problème, lourd d'implications, de la datation à assigner à ce texte non daté. W.C. Till proposait, d'après l'écriture, « etwa des VII. Jahrhunderts », date qui a été ensuite reprise sans plus de précision. Or le vII siècle n'est pas n'importe quel siècle ; c'est celui qui a vu, outre la parenthèse de l'occupation sassanide (619-629), la fin définitive de la domination byzantine en Égypte et son basculement sous la loi arabo-musulmane en 641-642. La portée institutionnelle et sociolinguistique de notre document n'est évidemment pas la même s'il doit être situé avant ou après 642. Peut-on être plus précis ?

L'approche paléographique n'autorise aucune certitude, tandis que la prosopographie ne permet aucun recoupement avec d'autres textes d'Edfou. Auronsnous plus de chance avec le vocabulaire institutionnel, en l'occurrence le nom de fonction $\check{\varepsilon} \kappa \delta 1 \kappa о \varsigma$ ? De prime abord, non : c'est une fonction dont les attestations vont au-delà de la conquête arabo-musulmane. Mais les occurrences après 642 sont d'une extrême rareté : les deux seules que pouvait citer B.R. Rees dans son étude sur le defensor civitatis en Égypte sont erronées $\left({ }^{22}\right)$. Le mot est néanmoins bien présent dans le $P$. Apoll. 46 (Edfou, $2^{\mathrm{e}}$ moitié du VII ${ }^{\mathrm{e}}$ siècle), où il est question d'une prise de garants en présence de l'évêque et du defensor pour des raisons de perception fiscale (1. 5), et dans SPP X 252 (Arsinoïte, vIII ${ }^{\mathrm{e}}$ siècle) $\left({ }^{23}\right)$,

(21) Voir leur liste dans CPR XVIIA, pp. 79-80 (y ajouter SB XVIII 13780).

(22) B.R. REES, «The defensor civitatis in Egypt», JJP 6 (1952), pp. 73-102, n. 97 : SB I 4694, 4 (Arsinoïte, mi-VII ${ }^{\mathrm{e}}$ siècle ?) repose sur une restitution hasardeuse de Wessely, corrigée depuis (BL VIII 313) ; P. KRU 88 (= P. Lond. I 87 descr. ; P. Lond. Copt. I 386 descr. ; pour le grec, = SB I 5599) est aussi à éliminer car résultant d'une mauvaise interprétation du mot nєk $\omega \mathrm{T}$ (voir d'ailleurs, REEs, loc. cit., p. 97, n. 170). La plus récente étude sur ce fonctionnaire, celle de FRAKES, Contra Potentium Iniurias [n. 11], s'arrête au début du ve siècle.

(23) Encore que le VII ${ }^{e}$ siècle ne soit pas à rejeter catégoriquement : l'écriture présente en effet des traits plus anciens que le $\mathrm{VIII}^{\mathrm{e}}$ siècle (comme le reconnaît F. MorELLI, P.Horak, p. 185 d'après la photo publiée par G. Rouillard, L'administration civile de l'Égypte byzantine [Paris, 1928²], p. 155). 
liste de personnes emprisonnées mentionnant un defensor anonyme (1. 18). Deux autres documents peuvent être allégués, mais leur datation après 642 n'est pas certaine $\left({ }^{24}\right)$. Le defensor s'est certainement maintenu après la Conquête (comme d'autres fonctionnaires ou magistrats byzantins), mais force est de reconnaître qu'il devient quasiment invisible et que les rares attestations qui nous soient parvenues ne permettent pas de confirmer que ses fonctions judiciaires aient survécu à la fin de la domination byzantine $\left({ }^{25}\right)$. Il est en outre totalement absent de la documentation copte d'époque arabe $\left({ }^{26}\right)$, silence qui serait d'autant plus étonnant que notre papyrus montre ce fonctionnaire comme acceptant d'être approché en copte.

Il nous semble donc plus vraisemblable que ce papyrus date d'avant la Conquête $\left({ }^{27}\right)$. Il montre que l'administration judiciaire s'était finalement ouverte à la langue copte en autorisant la rédaction de pétitions dans cette langue, du moins dans les procédures impliquant le defensor. Il n'est cependant pas certain que cette ouverture soit entièrement le fait des Byzantins. Une autre pétition, publiée elle aussi comme une lettre et assez similaire dans sa forme, quoique adressée par une veuve à un évêque, remonte, elle clairement, à l'époque sassanide (619-629) du fait d'allusions aux Perses $\left({ }^{28}\right)$. La conquête sassanide a pu, en coupant un temps l'Égypte de l'État byzantin hellénophone, encourager la population coptophone ne maîtrisant pas le grec à aspirer à une place plus affirmée du copte dans la vie publique et inciter une partie de ses élites à accéder à cette revendication ${ }^{29}$ ). Celle-ci remonte cependant à plus loin, comme le montrent les premiers arbitrages en copte du milieu du $\mathrm{VI}^{\mathrm{e}}$ siècle et les premiers documents juridiques de la fin du même siècle.

La plainte de Merkouria est la première pétition en copte adressée à un fonctionnaire. Gageons cependant que d'autres pourront encore être identifiées dans le vaste corpus des lettres coptes. En ébranlant définitivement le dogme de

(24) CPR XIV 17 (Arsinoïte, 652 ou 636 [l'éditrice préfère la première date]) ; SB I 4490 (Arsinoïte, 656 ou 641). Voir la discussion dans $C P R$ XIV 17, intr.

(25) Cf. G. FAnTONI, CPR XIV 17, 7 n. : « It is not known what legal or administrative functions the defensor civitatis retained in the Arab period because references to the $\varepsilon \kappa \delta 1 \kappa o \varsigma$ in the

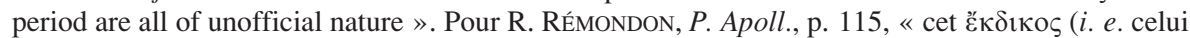
de $P$. Apoll. 46) [...] nous semble être l'héritier direct des $\varepsilon_{\kappa} \delta \delta$ เко byzantins », mais c'est surtout dans son rôle de magistrat municipal que Rémondon voit des continuités. Aucun des papyrus d'époque arabe n'évoque un rôle judiciaire du defensor.

(26) La seule occurrence enregistrée par Förster, WB, est d'époque byzantine ( $P$. Lond. V 1709 [Antinoopolis, 570]). On peut y ajouter l'endossement copte de $B G U$ IV 1094 (Hermoupolis, 525).

(27) Même si une datation du tout début de la période arabe ne nous semble pas impossible, ni priver ce papyrus de tout son intérêt sociolinguistique, les premières décennies du régime arabomusulman étant encore très tributaires de la situation institutionnelle précédente.

(28) SB Kopt. I 295 (Thèbes, époque sassanide).

(29) Cf. Fournet, Egyptian versus Greek [n. 3]. 
l'usage exclusif du grec pour les pétitions, elle jette des lumières nouvelles sur les institutions judiciaires du $\mathrm{VII}^{\mathrm{e}}$ siècle, mais montre surtout qu'il reste encore beaucoup à faire en la matière.

Université libre de Bruxelles (ULB)

Alain DelatTRE

École pratique des hautes études (EPHE), PSL

Collège de France

Jean-Luc FouRnET

École pratique des hautes études (EPHE), PSL 


\section{ANNEXE : LES COMPTES NOTÉS AU VERSO DE SB KOPT. IV 1709}

Une fois la pétition republiée ci-dessus périmée, le papyrus a été réutilisé par une cinquième main, peu habile, pour noter des comptabilités. Trois comptes sont écrits en des sens divers sur le verso de la pétition. Selon toute vraisemblance, le scribe a noté en premier lieu le compte E1, sur la moitié du verso restée vierge, qui offrait la surface la plus large. Il a ensuite rédigé les deux comptes suivants, l'un (E2) à $90^{\circ}$ par rapport à E1, entre ce dernier et l'adresse de la pétition (D), l'autre (E3), tête-bêche par rapport à E1, dans l'espace situé à droite de l'endossement (D) (voir supra, fig. 2).

Les trois comptes sont liés : treize personnes sont citées en E1 et en E2, dont onze sont mentionnées dans les deux comptes. Le compte E3 est d'ampleur moindre : il ne reprend que sept individus, qui correspondent aux sept derniers cités dans E2 et qui se retrouvent tous dans E1 ; les montants dans E2 et E3 semblent être en bonne partie les mêmes. On peut dès lors considérer soit que E3 constitue un compte préliminaire, reporté ensuite dans E2 et légèrement modifié, soit, puisque E3 est comme E2 pourvu d'un total, qu'il s'agit d'un sous-total des montants versés par les sept derniers contribuables, avec à nouveau une modification.

Les comptes E2 et E3 présentent en regard de chaque nom des sommes exprimées en carats. La situation est plus complexe dans E1, qui enregistre des paiements en solidi et en carats. Une précision apparaît aux lignes 2,4 et $8:$ après la mention du montant en nomismata, on trouve un second montant, précédé d'une sorte de $\chi$ ou de croix de multiplication. On voit mal quelle unité monétaire pourrait être ainsi désignée ; on pourrait penser à y voir un sigle pour $\pi \alpha \rho \alpha ́(1.2$, yo $(\mu i ́ \sigma \mu \alpha \tau \alpha) \alpha \mathrm{X} \beta$ signifierait « 1 solidus moins 2 carats »), même si cette graphie n'est pas attestée à notre connaissance. Le scribe de ces trois comptes semble plutôt mal à l'aise dans la rédaction comptable. Il maîtrise mal l'abréviation de vó $\mu 1 \sigma \mu \alpha$ et des fractions en général, dont la lecture est largement tributaire des totaux au bas de comptes E2 et E3 $\left({ }^{30}\right)$.

L'onomastique du document le rattache à la région d'Edfou (voir ci-dessous les commentaires aux noms Haroou et Pistôi). On relève quelques noms nouveaux, comme

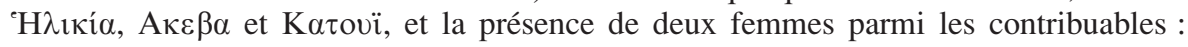
Rakhêl (E1, 7 ; E2, 24 ; E3, 31) et Hêlikia (E1, 11 ; E2, 21 ; E3, 35).

P. Vindob. Inv. K $950 \mathrm{v}$.

$8 \times 34,5 \mathrm{~cm}$ (FIG. 2)
Apollinopolis Anô (Edfou)

$1^{\text {re }}$ moitié du VII ${ }^{\mathrm{e}}$ siècle

$\mathrm{E} 1 \downarrow$ (à gauche de $\mathrm{D}$, écrit à $90^{\circ}$ )

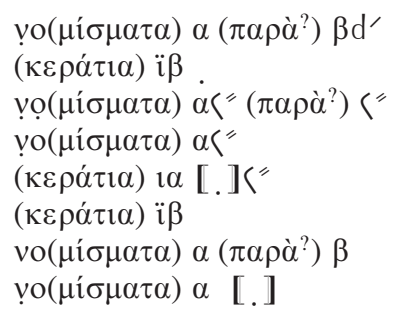

(30) Nous remercions Jean Gascou pour la lecture des fraction des lignes 1 et 3. 
$10 \Phi \imath \lambda o ́ \theta(\varepsilon \circ \varsigma)$

'Н $\lambda$ เкía

$\Pi \alpha \tau \varepsilon \rho \mu($ ov́ $) \theta($ เo $)$

$\Pi \alpha \varphi \varphi\left({\left.\text { vov́} \theta 1 o \varsigma^{?}\right)}^{2}\right.$

Avató $\lambda$ ios

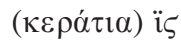

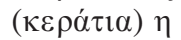
$(\kappa \varepsilon \rho \alpha \dot{\tau} 1 \alpha)$ ï
yo $(\mu i \sigma \mu \alpha \tau \alpha) \alpha "$
vo $(\mu i ́ \sigma \mu \alpha \tau \alpha) \alpha$

$\mathrm{E} 2 \rightarrow$ (à gauche de $\mathrm{D}$, écrit tête-bêche) :

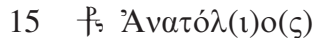

$\Pi \alpha \varphi\left(\right.$ voú $\left.\theta 1 \mathrm{c} \varsigma^{?}\right)$

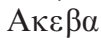

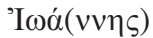

$\Sigma \varepsilon v o u ́ \theta(10 \varsigma)$

20 Kaт̣ovï

${ }^{ } \mathrm{H} \lambda \imath$ «ía

Чочк1

$\Phi 1 \lambda \circ \theta^{\prime}(\varepsilon \circ \varsigma)$

${ }^{\mathrm{P}} \mathrm{P} \alpha \chi \eta^{\prime} \lambda$

25 'Тккஸ́ $\beta$

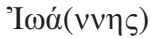

Apoov

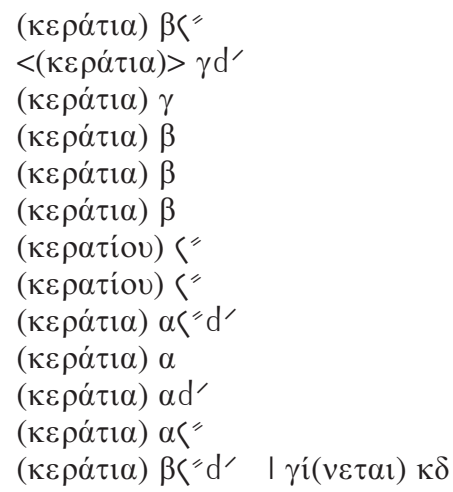

$\mathrm{E} 3 \downarrow$ (à droite de $\mathrm{D}$, écrit à $90^{\circ}$, tête-bêche par rapport à E1) :

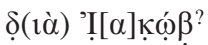
$\dot{\delta}(1 \grave{\alpha}){ }^{\prime} \mathrm{I} \omega \alpha \dot{(v \vee v o v)}$

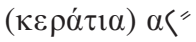

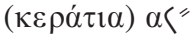
$\delta\left(\right.$ ià) ${ }^{\mathrm{e}} \mathrm{P} \alpha \chi \eta^{\prime} \lambda$

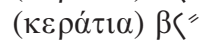
$\delta($ ià) Чоणкı

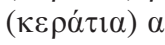
$\delta(\imath \grave{\alpha}) \Phi ı \lambda \circ \theta(\dot{\varepsilon} \circ v)$
(кератíov) ("

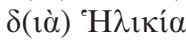
$\left[(\kappa \varepsilon \rho \alpha \dot{\tau} \tau \alpha) \alpha \zeta^{\prime \prime}\right] d^{\prime}$ ?

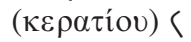

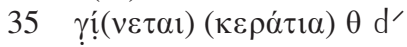

$30 \delta($ ì̀) Apoov

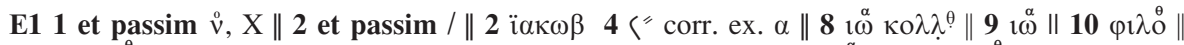

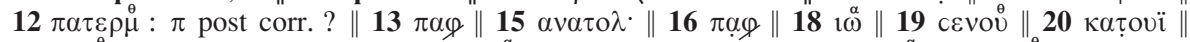

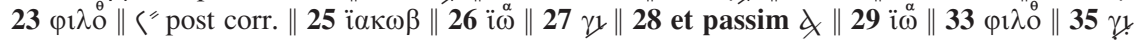

$\ll \quad \dagger$

Senouthios

Iakôb

Haroou

5 Pistôi

Psouki

Rakhêl

Iôannês, fils de Kollouthos

Iôannês

10 Philotheos

Hêlikia

Patermouthios
1 nom. moins? 2 1/4 (car.)

12 car.

$11 \frac{1}{2}$ nom. moins? $1 / 2$ (car.)

$11 / 2$ nom.

$11 \frac{1}{2}$ car.

12 car.

1 nom. moins? 2 (car.)

1 nom.

16 car.

8 car.

16 car. 
Paphnouthios?

Anatolios

E2

15 Anatolios

Paphnouthios?

Akeba

Iôannês

Senouthios

20 Katoui

Hêlikia

Psouki

Philotheos

Rakhêl

25 Iakôb

Iôannês

Haroou

E3

Par Iakôb?

Par Iôannês

30 Par Haroou

Par Rakhêl

Par Psouki

Par Philotheos

Par Hêlikia

35 Total : $91 \frac{1}{4}$ carats. »
$11 / 2$ nom.

$1 \mathrm{nom}$.
$21 / 2$ car.

$31 / 4$ car.

3 car.

2 car.

2 car.

2 car.

$1 / 2$ car.

$1 / 2$ car.

$1 \frac{1}{1 / 2} 1 / 4$ car.

1 car.

$11 / 4$ car.

$1 \frac{1}{2}$ car.

$2 \frac{1}{2} 1 / 4$ car. | Total : 24 (car.)

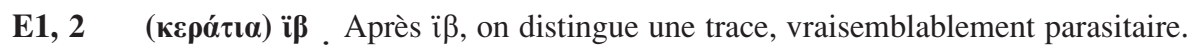

E1, 4 Apoov Le nom est attesté plusieurs fois dans la documentation grecque comme copte (www.trismegistos.org/name/6866). Pour l'étymologie de l'anthroponyme, cf. J.-L. FouRnET, «Quittances de loyer du topos d'apa Michel d'Antaiopolis », BASP 45 (2008), pp. 45-58, en part. pp. 50-52.

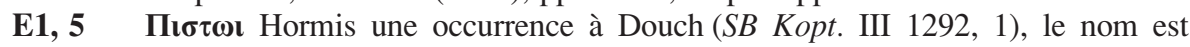
attesté exclusivement à Edfou (cf. www.trismegistos.org/name/11645 ; cf. aussi O. Edfou Ifao 93, 6 corrigé dans A. Delattre \& J.-L. FouRnET, « Les ostraca grecs et coptes d'Edfou. À propos d'une publication récente », ArchPF 57 [2011] pp. 79-98, en part. p. 94).

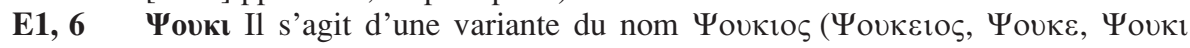
cf. www.trismegistos.org/name/13101 et 39044), attesté à l'époque byzantine principalement à Aphroditê, mais aussi dans le Panopolite ( $P$. Lond. V 1653, 36) et l'Heracléopolite ( $P$. Vind. Tand. 16, 3, 46).

E1, 11 'H $\boldsymbol{\lambda}$ ıкí $\boldsymbol{\alpha}$ L'anthroponyme est inconnu par ailleurs; il faut sans doute l'inter-

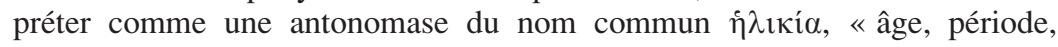

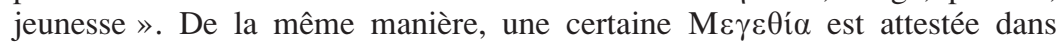
O. Edfou Ifao 68, 6 et $O$. Edfou II 316, 3 ; cf. R. DANIEL, « P.Oxy. XLI 2951 and O Edfou II 316 », ZPE 159 (2007), p. 70. 
E2, 18 Акв $\boldsymbol{\beta} \boldsymbol{\alpha}$ Le nom est inconnu par ailleurs.

E2, 20 Ka⿱宀ovï Le nom ne semble pas être attesté ailleurs dans la documentation. On trouve deux anthroponymes proches: Kavous (SB XX 16000, 13, 438)

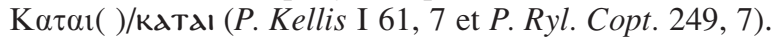

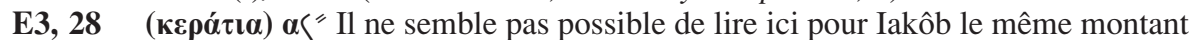
que dans E2.

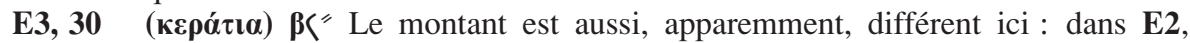
Haroou s'acquitte de la somme de $2 \frac{112}{1 / 4} \mathrm{car}$. 